\title{
Cell type-specific anti-cancer properties of valproic acid: independent effects on HDAC activity and Erk1/2 phosphorylation
}

\author{
Kamil Gotfryd, Galina Skladchikova, Eugene A Lepekhin, Vladimir Berezin, Elisabeth Bock, Peter S Walmod
}

\begin{abstract}
Background: The anti-epileptic drug valproic acid (VPA) has attracted attention as an anti-cancer agent.

Methods: The present study investigated effects of VPA exposure on histone deacetylase (HDAC) inhibition, cell growth, cell speed, and the degree of Erk1/2 phosphorylation in 10 cell lines (BT4C, BT4Cn, U87MG, N2a, PC12-E2, CSML0, CSML100, HeLa, L929, Swiss 3T3).

Results: VPA induced significant histone deacetylase (HDAC) inhibition in most of the cell lines, but the degree of inhibition was highly cell type-specific. Moreover, cell growth, motility and the degree of Erk $1 / 2$ phosphorylation were inhibited, activated, or unaffected by VPA in a cell type-specific manner. Importantly, no relationship was found between the effects of VPA on HDAC inhibition and changes in the degree of Erk1/2 phosphorylation, cell growth, or motility. In contrast, VPA-induced modulation of the MAPK pathway downstream of Ras but upstream of MEK (i.e., at the level of Raf) was important for changes in cell speed.
\end{abstract}

Conclusions: These results suggest that VPA can modulate the degree of Erk1/2 phosphorylation in a manner unrelated to HDAC inhibition and emphasize that changes in the degree of Erk1/2 phosphorylation are also important for the anti-cancer properties of VPA.

\section{Background}

Valproic acid (2-n-propylpentanoic acid, VPA) is a common anti-epileptic drug that is also used for the treatment of bipolar disorder, migraine and neuropathic pain [1]. Moreover, VPA can modulate several cancer-related processes, including angiogenesis, immunogenicity, and invasion, metastasis, differentiation, proliferation and apoptosis of cancer cells [2]. Recent clinical studies have demonstrated the chemotherapeutic efficacy of VPA for the treatment of several types of cancer, including acute myeloid leukemia, myelodysplastic syndromes [3], and solid breast and cervix tumors [4].

VPA is a histone deacetylase (HDAC) inhibitor that alters gene expression, thereby modulating processes such as cell growth, differentiation and apoptosis [5,6]. The drug is also known to modulate the activity of several intracellular enzymes, including mitogen-activated

\footnotetext{
* Correspondence: psw@sund.ku.dk

Protein Laboratory, Department of Neuroscience and Pharmacology, Faculty of Health Sciences, University of Copenhagen, Denmark
}

protein kinases (MAPKs), protein kinase $\mathrm{C}(\mathrm{PKC})$ and

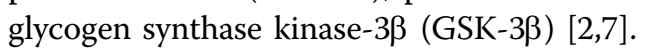

Several of the cellular processes modulated by VPA may be partially regulated by signaling through the MAPK pathway [8-10]. Signaling through this pathway is often initiated by activation of membrane-localized receptors, leading to activation of the GTPase Ras, and subsequent activation of the MAPK kinase kinase Raf, the MAPK kinases MEK1/2 and the MAPKs Erk1/2 [11]. Activated Erk1/2 phosphorylate targets in the cytosol and transcription factors in the nucleus [12]. Erk $1 / 2$ can also be activated Ras-independently by other upstream molecules, including protein kinase A (PKA) and PKC. Furthermore, cells express up to three Raf types (i.e., A-, B- and c-Raf), which are affected differently by upstream targets, thereby adding a further level of complexity to MAPK-mediated signaling $[13,14]$.

The anti-cancer effects of VPA are generally attributed to its HDAC inhibitory activity. However, these effects may also be partially caused by alterations in, for example, Erk1/2 activity. Interestingly, previous studies have 
shown cell type-specific effects of VPA, both on specific cellular processes, such as cell migration and proliferation [15], and on specific enzyme activities, including Erk $1 / 2$ activity $[16,17]$. The reasons for these cell typespecific effects, however, are unknown.

The aim of the present study was to investigate the relationships between VPA-induced changes in HDAC and Erk $1 / 2$ activities, and cell growth and motility. The results reveal striking cell type-specific differences in the responses to VPA. Moreover, the effects of VPA on cell growth, motility and the degree of Erk $1 / 2$ phosphorylation were not related to its effects on HDAC inhibition. In contrast, modulation of cell growth and motility was in some cell lines related to changes in the degree of Erk1/2 phosphorylation, which was regulated at the level of Raf.

\section{Methods \\ Cell cultures}

Cells were grown in a humidified atmosphere at $37^{\circ} \mathrm{C}$, $5 \% \mathrm{CO}_{2}$. The rat glioma cell lines BT4C and BT4Cn, the human glioma cell line U87MG (a gift from Dr. Nina Pedersen, Copenhagen University Hospital Rigshospitalet, Copenhagen, Denmark), the mouse neuroblastoma cell line N2a, the mouse adenocarcinoma cell lines CSML0 and CSML100, the human adenocarcinoma cell line HeLa, and the mouse fibroblastoid cell lines Swiss 3T3 and L929 were grown in Dulbecco's modified Eagle's medium (DMEM) supplemented with $10 \%(\mathrm{v} / \mathrm{v})$ heat-inactivated fetal calf serum (FCS), $2 \mathrm{mM}$ GlutaMAX, $100 \mathrm{U} / \mathrm{ml}$ penicillin, $100 \mu \mathrm{g} / \mathrm{ml}$ streptomycin and $2.5 \mu \mathrm{g} / \mathrm{ml}$ fungizone (all from Invitrogen, Taastrup, Denmark). Cells were dislodged with trypsin/ EDTA in modified Puck's saline (Invitrogen).

The rat pheochromocytoma cell line PC12-E2 was grown in DMEM supplemented with 5\% (v/v) FCS, 10\% (v/v) horse serum, $2 \mathrm{mM}$ GlutaMAX, $100 \mathrm{U} / \mathrm{ml}$ penicillin and $100 \mu \mathrm{g} / \mathrm{ml}$ streptomycin (Invitrogen). Cells were dislodged by tapping the culture flask.

\section{Cell transfection}

Stable transfection of L929 with the pGV16 vector encoding constitutively active rat H-Ras (G12V; a gift from Prof. Berthe Willumsen, Department of Biology, Faculty of Science, University of Copenhagen, Copenhagen, Denmark) was obtained using Lipofectin (Invitrogen). After transfection, cells were grown in medium containing $0.75 \mathrm{mg} / \mathrm{ml}$ geneticin (Invitrogen) for 3 weeks. Six geneticin-resistant clones with high $\mathrm{H}$ RasG12V expression were selected, propagated, pooled and used as stock for subsequent experiments. Ten randomly selected clones stably transfected with the empty pGV16 vector were selected, propagated, pooled and used as control cells.
Transient transfections of cells with the pGV16 vector encoding dominant-negative rat H-Ras $(\mathrm{S} 17 \mathrm{~N}$; a gift from Dr. Berthe Willumsen), the pRK5 vector encoding constitutively active rat MEK2 (S222E/S226E; a gift from Dr. Klaus Seedorf, Hagedorn Research Institute, Gentofte, Denmark), or the corresponding empty vectors were performed using FuGene 6 (Roche Diagnostics, Mannheim, Germany) or Lipofectin 2000 (Invitrogen). Transient transfections were performed as co-transfections with low amounts of the pEGFP-N1 vector (Clontech, Saint-Germain-en-Laye, France). Consequently, transfected cells were identified from their expression of enhanced green fluorescent protein. Cells were replated for subsequent experiments $\sim 24 \mathrm{~h}$ after transfection.

\section{VPA treatment}

A stock solution of 3 M VPA (Sigma-Aldrich, Copenhagen, Denmark) was prepared in dimethylsulfoxide (DMSO), and all assays were performed in the presence of $0.1 \%(\mathrm{v} / \mathrm{v})$ DMSO in the presence or absence of VPA.

\section{Immunoblotting}

Cells were plated in $60 \mathrm{~mm}$ culture dishes (Nunc, Roskilde, Denmark) at a density of 0.25-0.75 $\times 10^{6}$ cells/ dish and grown in medium containing 0-3 mM VPA for up to $48 \mathrm{~h}$. In experiments in which cells were exposed to a MEK inhibitor (PD98059; Cell Signaling, Danvers, MA), the compound was added to the cultures $1 \mathrm{~h}$ before cell lysis. Following incubation, cells were rinsed in ice-cold PBS and collected in radioimmunoprecipitation assay lysis buffer supplemented with Complete, EDTA-free Protease Inhibitor Cocktail (Roche Diagnostics) and Phosphatase Inhibitor Cocktail Set III (Calbiochem). Proteins were resolved by sodium dodecyl sulfate-polyacrylamide gel electrophoresis (SDS-PAGE) and transferred to Immobilon-P membranes (Millipore, Billerica, MA). The membranes were blocked in PBS containing $0.1 \%(\mathrm{v} / \mathrm{v})$ Tween -20 and $5 \%(\mathrm{w} / \mathrm{v})$ bovine serum albumin.

Following incubation with primary antibodies, all membranes were incubated with horseradish peroxidase-conjugated goat anti-rabbit antibody (Cell Signaling), visualized by chemiluminescence (SuperSignal West Dura Extended Duration Substrate, Pierce Biotechnology, Rockford, IL) using a GeneGnome (Syngene, Cambridge, UK), and quantified using the accompanying GenTools software.

For estimation of Erk $1 / 2$ phosphorylation, membranes were probed with anti-phospho-Erk1/2 (Thr202/Tyr204) antibody (Cell Signaling). After visualization, the membranes were stripped $\left(30 \mathrm{~min}, 50^{\circ} \mathrm{C}\right)$ in $62.5 \mathrm{mM}$ Tris-HCl, pH 6.8, 2\% (w/v) SDS and $50 \mathrm{mM}$ 1,4-dithioL-threitol, blocked, and reprobed with anti-pan-Erk1/2 antibody (Cell Signaling). Ras expression was 
determined using anti-H-, K- and N-Ras antibodies (Cell Signaling). Histone acetylation was determined using anti-acetyl histone H3 (Lys9/Lys14) antibody (Cell Signaling). Ras and histone $\mathrm{H} 3$ acetylation immunoblots were stripped and reprobed with anti-actin antibody (Sigma-Aldrich).

\section{Cell growth}

Cell growth was quantified from crystal violet stainings. $5 \times 10^{3}$ cells/well were plated in 96-well microwell plates (Nunc) and grown in medium containing 0-3 mM VPA for $48 \mathrm{~h}$, with each treatment replicated in 6 wells/ plate. Cells were fixed in $3.7 \%(\mathrm{v} / \mathrm{v})$ formalin and $1 \%(\mathrm{v} /$ v) methanol in PBS, and stained with $0.04 \%(w / v)$ crystal violet in $4 \%(\mathrm{v} / \mathrm{v})$ ethanol. Finally, the cell-bound crystal violet was solubilized using $1 \%(\mathrm{w} / \mathrm{v})$ SDS, and optical density was measured at $600 \mathrm{~nm}$.

\section{Measurement of individual cell motility}

$6-15 \times 10^{3}$ cells/well were plated in 6-well culture plates (Nunc) and grown in medium containing $0-3$ $\mathrm{mM}$ VPA. Time-lapse video-recordings were performed using a computer-assisted microscope workstation as previously described [18]. Simultaneous, sequential recordings from 8-20 microscopic fields/well were performed at 1-10 min intervals for $20-80 \mathrm{~min}$. Evaluation of individual cell motility was performed using the image processing software PRIMA (Protein Laboratory, Copenhagen University, Denmark). 50-200 cells from each well were tracked, and the data were used for the calculation of the mean-cell speed and the mean-squared cell displacement $\left(\left\langle d^{2}\right\rangle\right)$ as previously described [19].

\section{Statistics}

Results are expressed as mean \pm SEM, calculated on the basis of the number of experiments. Unless indicated otherwise, statistical analyses were performed on nonnormalized data using one-way repeated-measures analysis of variance (ANOVA) followed by the TukeyKramer multiple comparison test. Immunoblots were evaluated using the Wilcoxon signed rank test. Estimates of $I C_{25}$ and $I C_{50}$ values were based on the interpolation of data from log dose-response curves. Correlations were performed as Pearson correlations. All p-values refer to two-tailed calculations. "*** and ${ }^{* * * *}$ indicate $\mathrm{p}<0.05,0.01$ and 0.001 , respectively.

\section{Results}

Effects of VPA on the degree of Erk1/2 phosphorylation and histone $\mathrm{H} 3$ acetylation

Erk1/2 activity was investigated in 10 cell lines, including BT4C, BT4Cn, U87MG, N2a, PC12-E2, CSML0, CSML100, HeLa, Swiss 3T3 and L929, by estimating the degree of Erk1/2 phosphorylation by immunoblotting. In the absence of VPA, BT4C, BT4Cn, U87MG, and PC12-E2 exhibited high Erk1/2 phosphorylation levels. L929, Swiss 3T3 and CSML0 exhibited intermediate levels, and N2a, CSML100 and HeLa exhibited low levels (Figure 1a). Figure $1 \mathrm{~b}$ and $1 \mathrm{c}$ show the relative changes in the degree of Erk $1 / 2$ phosphorylation in the respective cell lines in response to VPA ( $3 \mathrm{mM}, 48 \mathrm{~h}$ ). Erk $1 / 2$ phosphorylation was significantly decreased in CSML0 and L929, significantly increased in CSML100, BT4Cn and N2a, and not significantly affected in BT4C, U87MG, PC12-E2, HeLa and Swiss 3T3.

VPA is a known HDAC inhibitor. Figure 1d shows the degree of acetylation of histone $\mathrm{H} 3$ in the 10 cell lines under control conditions determined by immunoblotting. All cell lines exhibited low acetylation levels under control conditions (Figure 1d).

VPA significantly increased the degree of acetylation in 8 of the 10 cell lines (Figure 1e and 1f). The changes in acetylation ranged from an approximately three-fold increase (U87MG) to an 13-fold increase (BT4C; Figure $1 \mathrm{e})$.

In conclusion, VPA has highly cell type-specific effects on the degree of HDAC inhibition and on changes in the degree of Erk1/2 phosphorylation. Furthermore, the effects of VPA on the degree of Erk1/2 phosphorylation and HDAC inhibition were not related to the degree of Erk1/2 phosphorylation and acetylation in the absence of the drug. Finally, no relationship was found between the effects of VPA on HDAC inhibition and the degree of Erk1/2 phosphorylation. Thus, VPA-induced changes in the degree of Erk1/2 phosphorylation cannot be explained by the effects of the drug on HDAC activity, although HDAC inhibition may contribute to the observed effects.

\section{Effects of VPA on cell growth}

Figure 2 shows that VPA dose-dependently changed the growth of all investigated cell lines. Nine cell lines exhibited $\mathrm{IC}_{25}$ values ranging from 0.43 to $1.83 \mathrm{mM}$ (Table 1), whereas the growth of a single cell line, U87MG, was significantly elevated upon exposure to $0.75 \mathrm{mM}$ VPA.

A correlation analysis of the data presented in Figure 1 and 2 revealed no significant correlations between the effects of VPA on HDAC inhibition and cell growth or between the degree of Erk1/2 phosphorylation and cell growth (Figure S4). However, four of the five cell lines that did not demonstrate significant changes in the degree of Erk1/2 phosphorylation in response to VPA (Figure 1b) did not have $\mathrm{IC}_{50}$ values for growth within the tested concentration range (below $3 \mathrm{mM}$; Table 1), whereas all five cell lines demonstrating significant changes in the degree of Erk1/2 phosphorylation in 


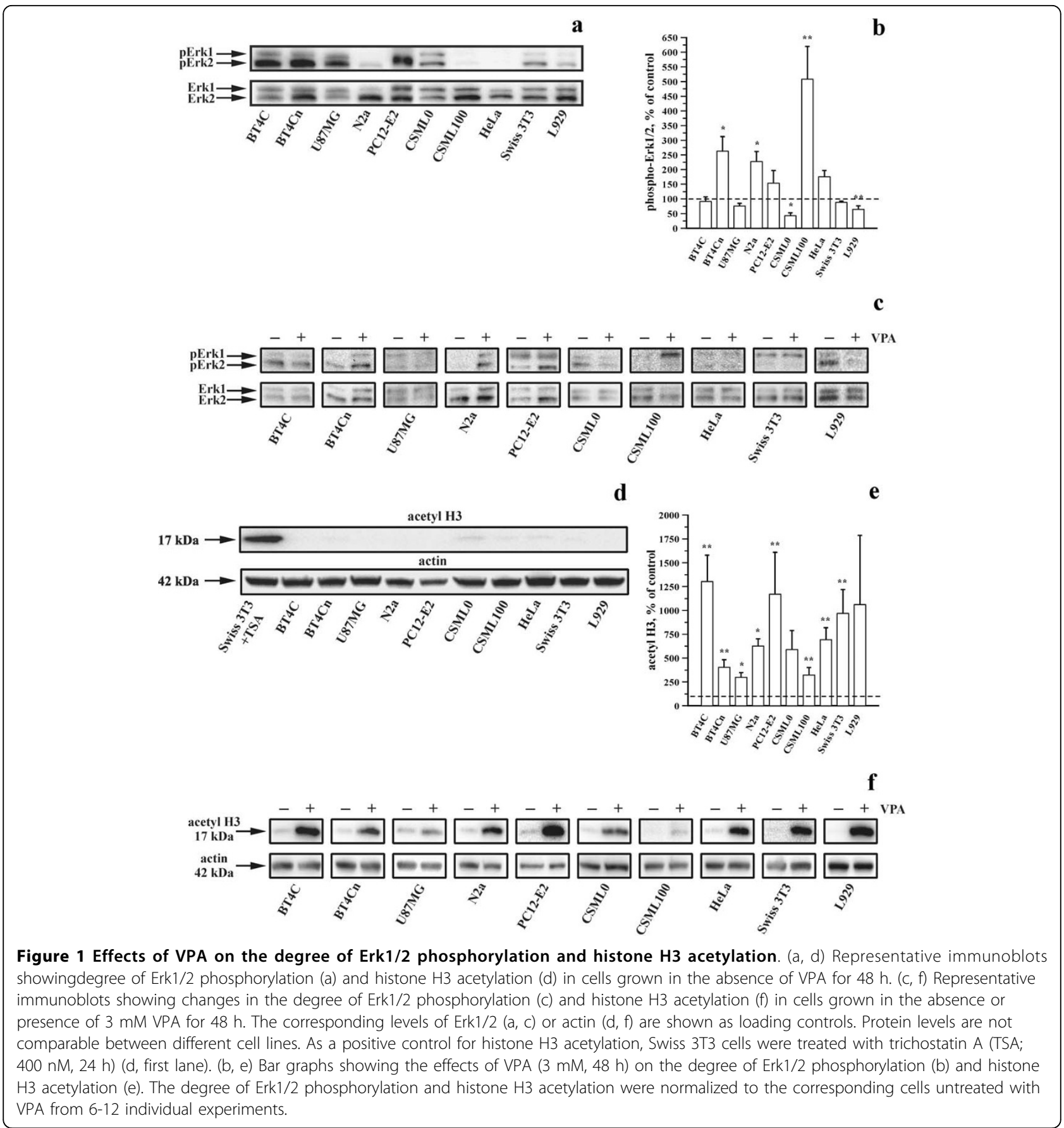

response to VPA had $\mathrm{IC}_{50}$ values for growth below $3 \mathrm{mM}$. These results demonstrate a significant relationship between the effects of VPA o the degree of Erk1/2 phosphorylation and cell growth $(\mathrm{p}<0.05$, Fisher's exact test).

In conclusion, VPA induced highly cell type-specific effects on cell growth. Furthermore, no apparent relationship was observed between the effects of VPA on HDAC inhibition and cell growth, whereas VPA was likely to affect the growth of a given cell type if it affected the degree of Erk $1 / 2$ phosphorylation in that cell type at physiologically relevant concentrations.

\section{Effects of VPA on individual cell motility and the degree of Erk1/2 phosphorylation in L929 cells}

Erk1/2 are known to regulate cell motility [20]. In L929 cells, VPA inhibits the degree of Erk1/2 phosphorylation (Figure 1) and cell speed [21]. Therefore, a potential 

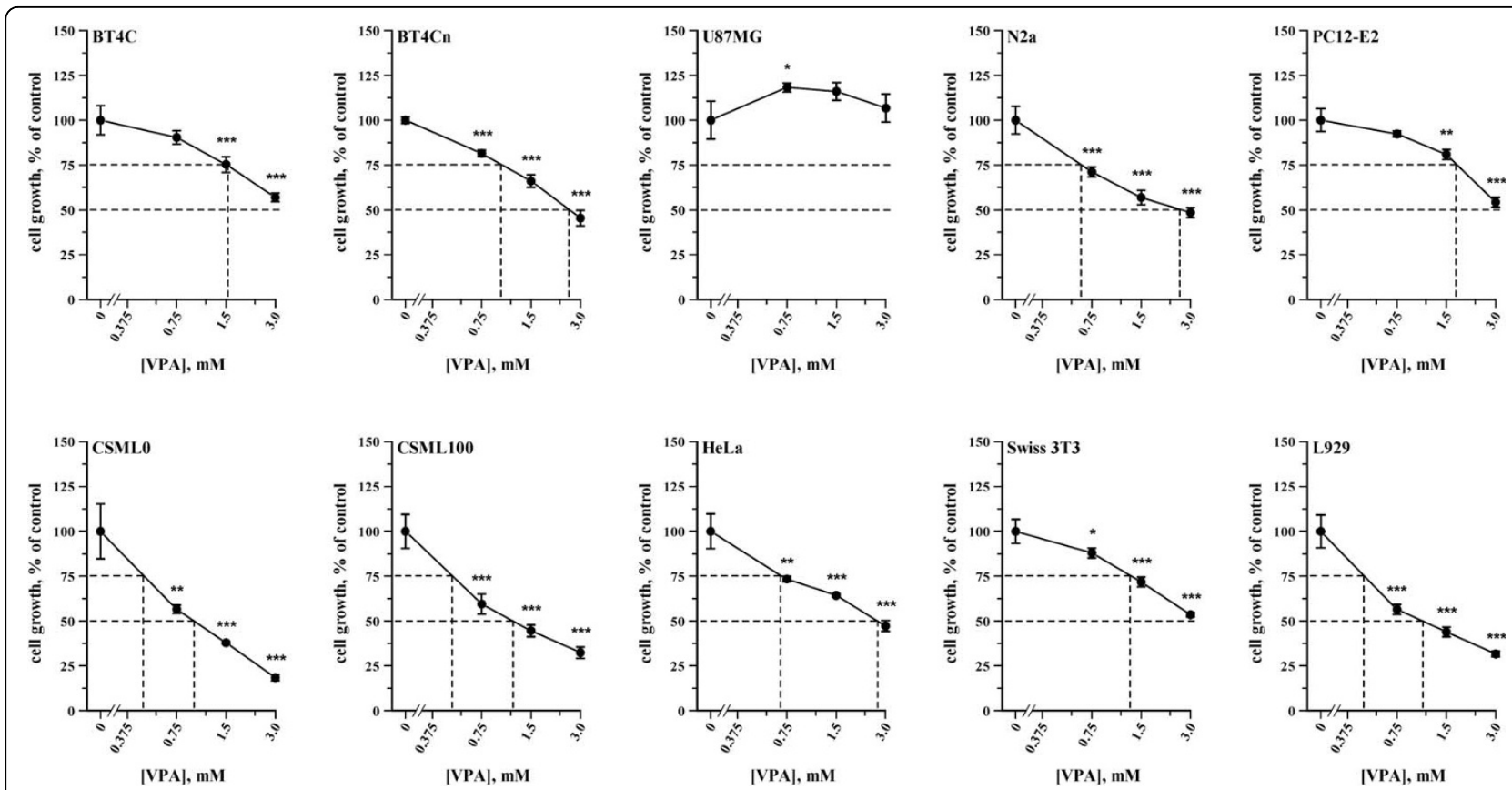

Figure 2 Effects of VPA on cell growth. Dose-response curves showing the effects of VPA on cell growth. Cells were exposed to VPA (0-3 mM, $48 \mathrm{~h}$ ) before growth was determined using crystal violet staining. Results were normalized to the corresponding cells untreated with VPA from four independent experiments.

relationship between the effects of VPA on the degree of Erk1/2 phosphorylation and cell motility was investigated further in L929 cells.

Figure 3a shows time-dependent effects of $3 \mathrm{mM}$ VPA on the mean-cell speed of L929 cells. The speed was significantly reduced after $1 \mathrm{~h}$ exposure to the drug. After 24-48 h exposure, further reductions in cell speed were observed. Figure $3 \mathrm{~b}$ shows data from the timeresponse experiments, in which VPA was directly added to the culture medium during the motility recordings. Using this approach, a reduction in cell displacement

Table 1 IC $_{25}$ and IC 50 values for the effects of VPA on cell growth

\begin{tabular}{ccccc}
\hline Cell Line & \multicolumn{2}{c}{$\boldsymbol{I C}_{\mathbf{2 5}}{ }^{\mathrm{a}}(\mathbf{m M})$} & \multicolumn{2}{c}{$\boldsymbol{I C}_{\mathbf{5 0}}{ }^{\mathrm{a}}(\mathbf{m M})$} \\
\hline BT4C & 1.52 & $(1.12-1.84)$ & $>3.0$ & - \\
BT4Cn & 1.06 & $(0.95-1.20)$ & 2.67 & $(2.37-2.98)$ \\
U87MG & $>3.0$ & - & $>3.0$ & - \\
N2a & 0.65 & $(0.54-0.72)$ & 2.73 & $(2.11-3.19)$ \\
PC12-E2 & 1.83 & $(1.67-1.99)$ & $>3.0$ & - \\
CSML0 & 0.43 & $(0.24-0.53)$ & 1.01 & $(0.94-1.08)$ \\
CSML100 & 0.46 & $(0.32-0.58)$ & 1.22 & $(0.98-1.41)$ \\
HeLa & 0.70 & $(0.62-0.75)$ & 2.75 & $(2.54-3.01)$ \\
Swiss 3T3 & 1.35 & $(1.22-1.47)$ & $>3.0$ & - \\
L929 & 0.43 & $(0.32-0.51)$ & 1.13 & $(0.97-1.29)$ \\
\hline
\end{tabular}

$I C_{25}$ and $I C_{50}$ values for the effects of VPA on cell growth calculated from interpolations of data. a: Numbers in parentheses indicate interpolated values for $I_{25}$ or $I C_{50} \pm$ SEM (based on four independent experiments). was observed for VPA-treated cells $\sim 20$ min after drug addition compared with control cultures. These observations demonstrate that VPA caused biphasic, time-related inhibition of the cell speed of L929 cells, characterized by an initial, rapid inhibition followed by a further inhibition at later time-points.

Figure $3 \mathrm{c}$ and $3 \mathrm{e}$ show time-response effects of VPA on the degree of Erk $1 / 2$ phosphorylation. A $1 \mathrm{~h}$ exposure to $3 \mathrm{mM}$ VPA significantly decreased the degree of Erk1/2 phosphorylation. After 6 and $24 \mathrm{~h}$, the degree of Erk1/2 phosphorylation decreased further. Figure $3 \mathrm{~d}$ and $3 \mathrm{f}$ show dose-response curves of the effects of VPA on Erk $1 / 2$ phosphorylation. VPA dose-dependently inhibited the degree of Erk1/2 phosphorylation, with estimated $\mathrm{IC}_{25}$ and $\mathrm{IC}_{50}$ values of $\sim 0.24$ and $0.58 \mathrm{mM}$, respectively. A comparison of Figure 3a and 3d revealed that the time-course of the effects of VPA on the degree of Erk1/2 phosphorylation resembled that observed for the inhibition of cell speed, suggesting that VPA partially modulated cell speed through modulation of the degree of Erk1/2 phosphorylation.

Following the studies of L929 cells, the motility of the remaining nine cell lines was investigated. Figure 4 shows that VPA significantly and dose-dependently inhibited the speed of N2a, U87MG and PC12-E2 cells, stimulated the speed of BT4Cn cells, and did not affect the speed of the remaining five cell lines (BT4C, CSML0, CSML100, HeLa and Swiss 3T3). 


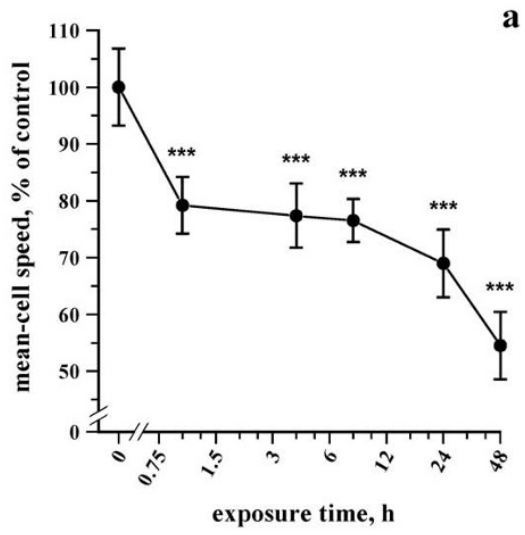

c
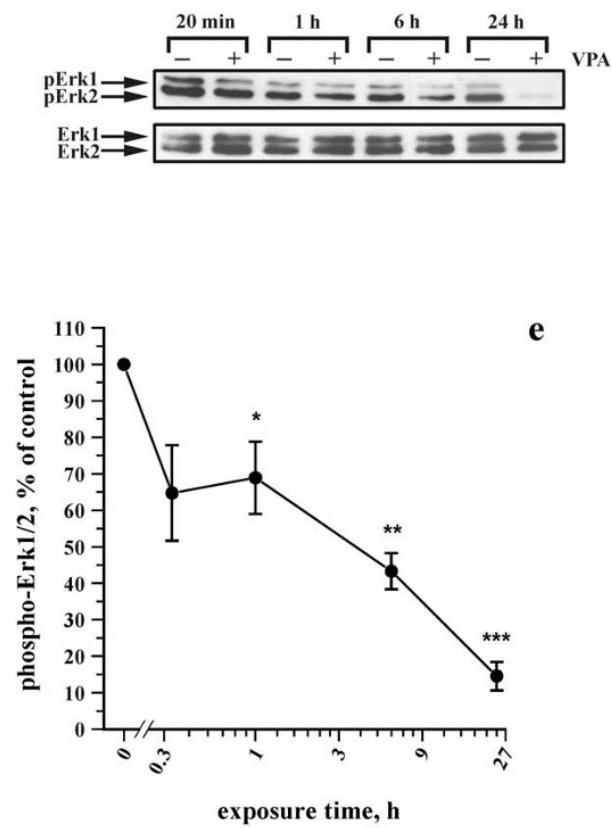

$\mathbf{e}$

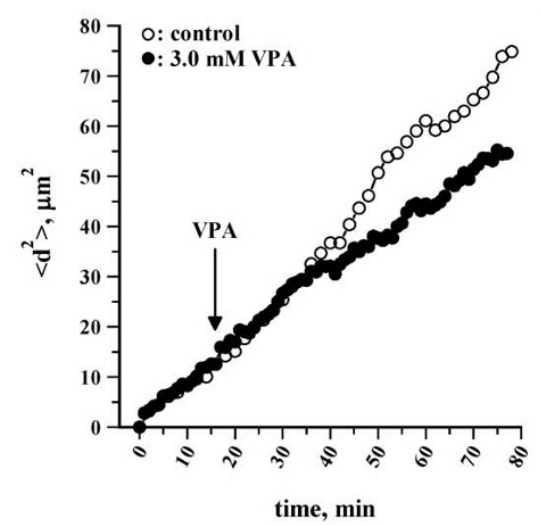

b

d
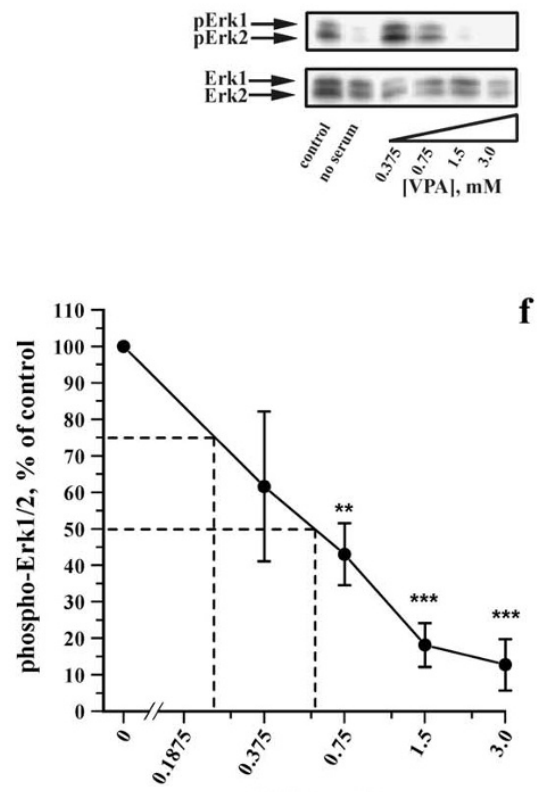

[VPA], $\mathrm{mM}$

Figure 3 Effects of VPA on individual cell motility and the degree of Erk1/2 phosphorylation in L929 cells. (a) Time-response curve showing the effects VPA on mean-cell speed of L929 cells exposed to 3 mM VPA. Cells were recorded for 20 min at 2 min intervals. The meancell speed of the individual cells was normalized to the corresponding cells untreated with VPA from four to seven independent experiments. (b) Time-response curve from a representative experiment showing the effect of VPA on mean-squared displacement $(<d 2>)$. At the indicated timepoint, VPA was added to the cell culture to a final concentration of $3 \mathrm{mM}$. Control (open circle) and VPA-treated (solid circle) cells were recorded at 2 and 1 min intervals, respectively. (c-f) Effects of VPA on the degree of Erk1/2 phosphorylation in L929 cells. (c, e) Representative immunoblots showing the degree of Erk1/2 phosphorylation in cells grown in the presence or absence of $3 \mathrm{mM} \mathrm{VPA} \mathrm{for} 20 \mathrm{~min}$ to $24 \mathrm{~h}$ (c) or the presence or absence of 0-3 mM VPA for $24 \mathrm{~h}$ (e). The corresponding levels of Erk $1 / 2$ are shown as loading controls. (d, f) Time- and doseresponse graphs showing the effects of $3 \mathrm{mM}$ VPA for 0-24 h (d) or 0-3 mM VPA for $24 \mathrm{~h}$ ( $\mathrm{f}$ ) on the degree of Erk1/2 phosphorylation, which was normalized to the corresponding cells untreated with VPA from four to five individual experiments.

Effects of VPA on individual cell motility by modulation of signal transduction upstream of Erk1/2

The GTPase Ras is an upstream activator of the MAPK pathway [11]. To determine whether VPA modulated the MAPK pathway upstream or downstream of Ras, the effects of VPA were investigated in L929 cells expressing constitutively active Ras (caRas). Figure 5a shows micrographs of L929 cells not expressing or expressing caRas and grown in the presence or absence of VPA. Control-transfected cells adopted a stellate phenotype with an increased area when treated with VPA (Figure 5a, two leftmost images), whereas caRasexpressing cells were more round and loosely attached (Figure 5a, two topmost images), a typical phenotype of 


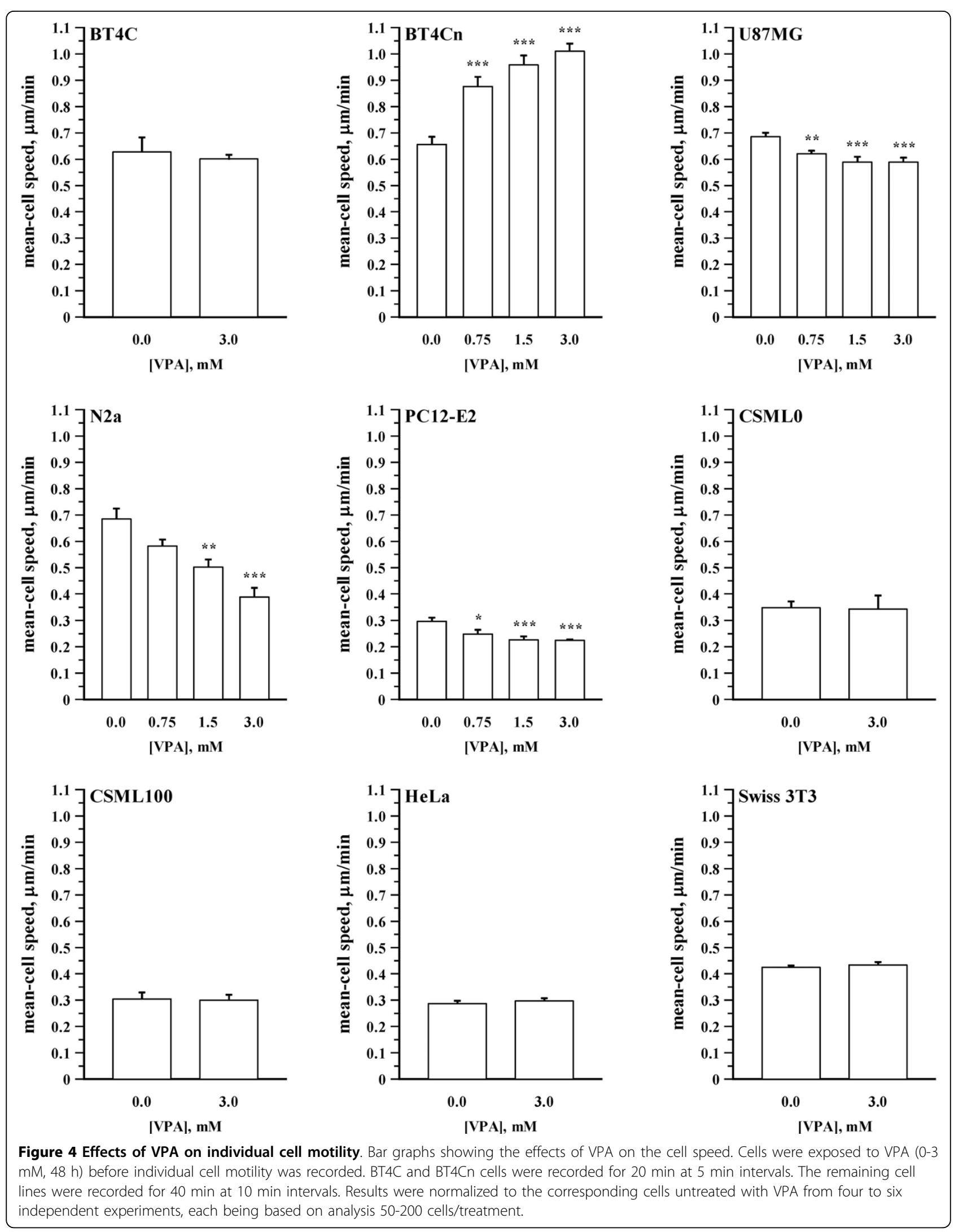




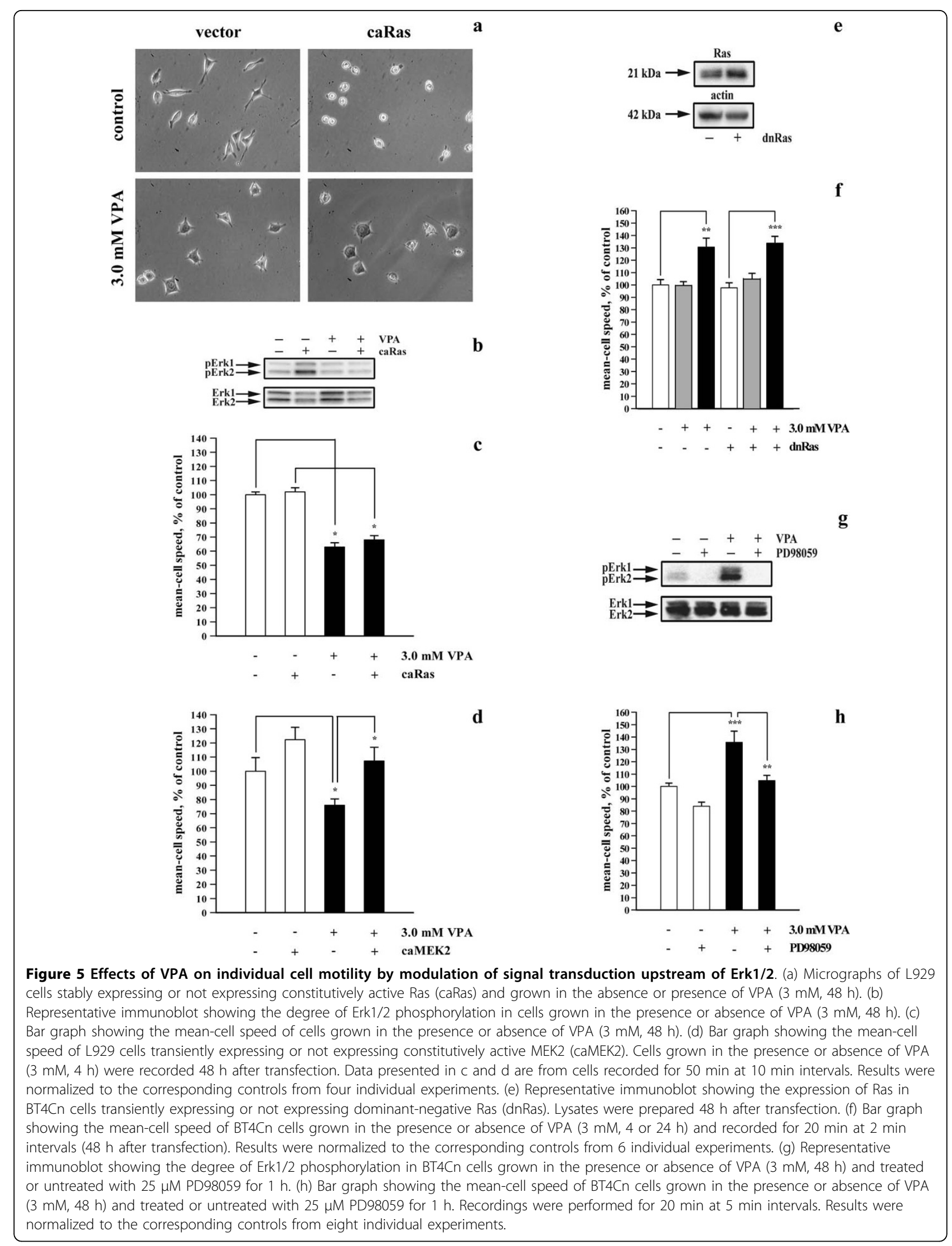


Ras-transformed cells [22]. However, exposure of caRasexpressing cells to VPA caused them to adopt a phenotype similar to control-transfected cells treated with VPA (Figure 5a, two rightmost images). The immunoblot presented in Figure 5b shows that L929 cells expressing caRas exhibited higher degrees of Erk $1 / 2$ phosphorylation than control-transfected cells when grown in the absence of VPA (Figure 5b, lanes 2 and 1). However, VPA caused equally strong inhibition of Erk $1 / 2$ phosphorylation in control- and caRastransfected cells (Figure 5b, lanes 3 and 4 ). These results demonstrate that exposure to VPA reversed the effects of caRas expression on L929 cell morphology and the degree of Erk1/2 phosphorylation. Cells expressing caRas did not move significantly faster than control-transfected cells when grown in the absence of VPA (Figure 5c). The speed of control- and caRas-transfected cells was similarly inhibited in the presence of VPA (Figure 5c).

Figure 5d shows the effects of VPA exposure on the speed of L929 cells expressing constitutively active MEK2 (caMEK2). Cells expressing caMEK2 did not move significantly faster than control-transfected cells grown in the absence of VPA (Figure $5 \mathrm{~d}, \mathrm{p}<0.063$ ). However, in the presence of VPA, caMEK2-expressing cells moved significantly faster than control-transfected cells exposed to VPA (Figure 5d, p < 0.029). Moreover, the speed of caMEK2-expressing cells was not significantly reduced after exposure to VPA (Figure $5 \mathrm{~d}$, column 4 vs. column 2).

To determine whether VPA affected the speed of BT4Cn cells through effects on the degree of Erk1/2 phosphorylation, the effects of VPA were investigated in BT4Cn cells expressing dominant-negative Ras (dnRas). The expression of dnRas was verified by immunoblotting using a polyclonal anti-Ras antibody. As shown in Figure 5e, Ras immunoreactivity was increased in cells transfected with the dnRas expression vector, indicating that the cells expressed dnRas. Figure $5 \mathrm{f}$ shows that exposure of BT4Cn cells to $3 \mathrm{mM}$ VPA for $24 \mathrm{~h}$, but not $4 \mathrm{~h}$, significantly increased the speed of both control- and dnRas-transfected cells. Thus, the expression of dnRas was not able to prevent the VPA-induced increase in the speed of BT4Cn cells.

Figure $5 \mathrm{~g}$ shows an immunoblot of BT4Cn cells treated or untreated with VPA and/or the MEK inhibitor PD98059. As expected, VPA increased the degree of Erk1/2 phosphorylation compared with untreated cells (Figure 5g, lanes 1 and 3). However, this stimulation was prevented by treating the cells with PD98059 (Figure $5 \mathrm{~g}$, lane 4). Similarly, treatment of BT4Cn cells with VPA increased the cell speed compared with untreated cells (Figure 5h, columns 1 and 3). However, the VPAinduced increase of the cell speed was completely prevented by PD98059 (Figure 5h, column 3 vs. column 4 ), which reduced the speed of VPA-treated cells to the level of control cells untreated with VPA and PD98059 (Figure 5h, columns 1 and 4). The same concentration of PD98059 did not significantly reduce the speed of cells untreated with VPA (Figure 5h, columns 1 and 2).

In conclusion, the data presented in Figure 5a-h demonstrate that VPA in L929 and BT4Cn cells modulates cell motility, cell morphology and the degree of Erk $1 / 2$ phosphorylation by altering signaling through the MAPK pathway downstream of Ras but upstream of MEK (i.e., at the level of Raf).

\section{Discussion}

The observation that VPA is an HDAC inhibitor $[6,23]$ has spurred numerous studies demonstrating that VPA possesses anti-cancer properties in vitro and in vivo $[2,5,24]$. However, VPA affects the activities of several enzymes and signal transduction pathways, and the mechanisms underlying the anti-cancer properties of VPA are not well characterized.

Consistent with earlier studies [25,26], we demonstrated that the degree of VPA-induced histone H3 acetylation was highly cell type-specific. Moreover, we found that the effect of VPA on the degree of Erk1/2 phosphorylation was highly cell type-specific. This observation is in contrast to the general notion that VPA, as demonstrated in several studies [17,27-30], activates Erk1/2, although inhibition has also been reported [16].

HDAC inhibitors can inhibit Erk1/2 activity [31,32]. However, consistent with recent studies [29,33], no relationship was observed between HDAC inhibition and the corresponding changes in the degree of Erk $1 / 2$ phosphorylation (Additional file 1, Figure S4). Therefore, effects of VPA on the degree of Erk1/2 phosphorylation and HDAC inhibition seem to be independent responses that, subsequently, may modulate biological processes such as cell growth or motility through independent mechanisms.

VPA-induced HDAC inhibition can hypothetically affect cell motility. Thus, VPA has in some [34], but not all [35], studies been shown to inhibit HDAC6, an enzyme known to modulate cell motility [36]. Likewise, VPA-induced HDAC inhibition can hypothetically affect cell growth. However, we did not find any correlations between the effects of VPA on HDAC activity and cell motility or growth (Additional file 1, Figure S4).

Erk1/2 activity controls multiple processes, including cell cycle progression, and cell growth, motility and survival [37]. Therefore, VPA-induced changes in Erk1/2 activity can hypothetically affect cell growth and motility. Hence, we focused our attention on the possible relationship between VPA-induced changes in Erk1/2 activity, cell growth and motility. 
Cell growth can be inhibited by both a sustained increase and decrease in Erk1/2 activity [37]. Therefore, no general correlation was found between the effects of VPA on the degree of Erk1/2 phosphorylation and cell growth (Additional file 1, Figure S4). However, cell lines demonstrating significant changes in the degree of Erk1/2 phosphorylation in response to VPA, generally had lower $\mathrm{IC}_{50}$ values for growth than cell lines with unaffected degrees of Erk1/2 phosphorylation (pERK-change, $\mathrm{p}<$ 0.05 and growth $\mathrm{IC}_{50}>3 \mathrm{mM}, 0$ cell lines; pERK-change, $\mathrm{p}<0.05$ and growth $\mathrm{IC}_{50}<3 \mathrm{mM}, 5$ cell lines; pERKchange, $\mathrm{p}>0.05$ and growth $\mathrm{IC}_{50}<3 \mathrm{mM}$, 4 cell lines; pERK-change, $\mathrm{p}>0.05$ and growth $\mathrm{IC}_{50}>3 \mathrm{mM}, 1$ cell line; $\mathrm{p}<0.05 ; \chi^{2}$ and Fisher's exact test). Therefore, we hypothesize that the modulation of the degree of Erk $1 / 2$ phosphorylation by VPA is of central importance for drug-mediated inhibition of cell inhibition.

We originally demonstrated VPA to inhibit the cell speed [21]. However, consistent with later studies $[15,38,39]$, the present study shows that the effects of VPA on the cell speed are highly cell type specific. Interestingly, a time-response of the VPA-induced change in L929 mean-cell speed exhibited a biphasic response, with a significant reduction detectable already after $\sim 20$ min followed by a further decrease after 24-48 h. Hence, the initial, rapid response must be independent of alterations in gene transcription, whereas the changes at later time-points may be the result of alterations in gene transcription.

The Ras-MAPK pathway regulates cell motility both independent of, and as a result of, changes in gene transcription [8]. However, Ras-MAPK signaling can affect different cell types differently. For example, VPA increased the degree of Erk1/2 phosphorylation in BT4Cn and N2a cells. However, BT4Cn cells maintained a de-differentiated phenotype, and exhibited an increase in both lamellipodia (Additional file 1, Figure S2 and Table S1) and the cell speed, whereas N2a cells, known to differentiate in response to a sustained increase in Erk1/2 activity [40], consequently demonstrated a decrease in the cell speed when exposed to VPA. Therefore, a direct correlation between changes in the degree of Erk $1 / 2$ phosphorylation and the cell speed is not to be expected and was not observed (Additional file 1, Figure S4). Nevertheless, a relationship was found since both L929 and BT4Cn cells demonstrated opposite effects with respect to changes in the degree of Erk1/2 phosphorylation and cell speed in response to VPA. Moreover, in both cell lines the effect of the drug on the Ras-MAPK pathway could be observed at a position downstream of Ras but upstream of MEK (i.e., at the level of Raf). This observation is consistent with a previous study in which abrogation of Ras signaling by preventing the farnisylation of the protein did not affect VPAmediated activation of Erk1/2 in endothelial cells [29].
Raf exists in three isoforms, A-, B- and c-Raf [13], which respond differently to Ras-independent upstream activators. PKA can stimulate the activity of B-Raf but inhibits the activity of c-Raf [41], which instead can be activated by PKC [14]. Consequently, cell type-specific effects of VPA on the degree of Erk1/2 phosphorylation may be partially explained by cell type-specific differences in the expression of Raf isoforms. An analysis of Raf expression revealed that all three Raf isoforms were expressed in all 10 investigated cell lines, although at highly variable levels (Additional file 1, Figure S3). However, no apparent relationship was found between the expression of the respective Raf isoforms and the observed changes in the degree of Erk $1 / 2$ phosphorylation in response to VPA. Notably, constitutively activated B- and c-Raf mutations are frequently observed in human cancers [42]. However, the analysis of Raf expression did not include studies of Raf mutations, and therefore the possibility that the expression of mutated Raf isoforms can contribute to the observed results cannot be excluded.

VPA has been suggested to be a possible chemotherapeutic drug for the treatment of gliomas $[4,5]$. However, consistent with recent observations [43], we found an increase in the growth of the human glioma U87MG at a physiologically relevant concentration of VPA. Moreover, the cell speed of the malignant glioma BT4Cn was profoundly increased in response to VPA. These observations suggest that VPA should be used with caution for the treatment of gliomas.

\section{Conclusions}

In conclusion, we demonstrate that VPA exposure induced considerable cell type-specific effects on HDAC inhibition, Erk1/2 phosphorylation, cell growth and motility. Furthermore, Erk1/2 phosphorylation, cell growth and motility are modulated independently of the degree of HDAC inhibition. In contrast, VPA affects signaling through the MAPK pathway at the level of Raf, thereby modulating cell growth and motility. These results suggest that the cell type-specific effects of VPA on the activity of Erk1/2 are important in relation to the use of VPA as an anti-cancer drug.

\section{Additional material}

\footnotetext{
Additional file 1: Supplementary methods. Supplementary references. Supplementary Table 1: Effects of VPA on cell morphology.

Supplementary figure legends. Supplementary figure 1: Effects of VPA on individual cell motility. (9 graphs) Supplementary figure 2: Effects of VPA on cell morphology. (20 micrographs) Supplementary figure 3: Analysis of A-, B-, and c-Raf expression. (3 agarose gels, 4 western blots) Supplementary figure 4: Relationships between changes in the degree of Erk1/2 phosphorylation, cell growth, cell speed, and histone $\mathrm{H3}$ acetylation in response to VPA treatment. (5 graphs)
} 


\section{List of abbreviations}

caMEK2: constitutively active MEK2; caRas: constitutively active Ras; DMEM: Dulbecco's modified Eagle's medium; DMSO: dimethylsulfoxide; dnRas:

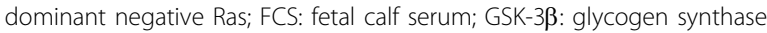
kinase-3 $\beta$; HDAC: histone deacetylase; MAPK: mitogen-activated protein kinase; PKA: protein kinase A; PKC: protein kinase C; SDS-PAGE: sodium dodecyl sulfate-polyacrylamide gel electrophoresis; VPA: valproic acid.

\section{Acknowledgements}

This work was kindly supported by "The Danish Research School in Molecular Cancer Research (MCRS)", "Fabrikant Einar Willumsens Mindelegat", "Fonden Victoria og Henry Andersens Legat", "Th. Maigaards Eftf. Fru Lily Benthine Lunds Fond af d. 1. juni 1978", "Civilingeniør Bent Bøgh og hustru Inge Bøghs Fond", "Else og Mogens Wedell-Wedellsborgs Fond and "Anders Hasselbalchs Fond til Leukæmiens Bekæmpelse".

\section{Authors' contributions}

EAL made the Ras-transfected cell lines. KG, GS and PSW made all experiments. KG, GS, VB, EB, and PSW participated in the study design. PSW and $\mathrm{KG}$ wrote the manuscript. All authors read and approved the final manuscript.

\section{Competing interests}

The authors declare that they have no competing interests.

Received: 14 January 2010 Accepted: 21 July 2010

Published: 21 July 2010

\section{References}

1. Johannessen CU, Johannessen SI: Valproate: past, present, and future. CNS Drug Rev 2003, 9:199-216.

2. Kostrouchova M, Kostrouch Z, Kostrouchova M: Valproic acid, a molecular lead to multiple regulatory pathways. Folia Biologica (Praha) 2007, 53:37-49.

3. Kuendgen A, Gattermann N: Valproic acid for the treatment of myeloid malignancies. Cancer 2007, 110:943-954

4. Duenas-Gonzalez A, Candelaria M, Perez-Plascencia C, Perez-Cardenas E, de la Cruz-Hernandez E, Herrera LA: Valproic acid as epigenetic cancer drug: preclinical, clinical and transcriptional effects on solid tumors. Cancer Treatment Reviews 2008, 34:206-222.

5. Michaelis M, Doerr HW, Cinatl J Jr: Valproic acid as anti-cancer drug. Current Pharmaceutical Design 2007, 13:3378-3393.

6. Phiel CJ, Zhang F, Huang EY, Guenther MG, Lazar MA, Klein PS: Histone deacetylase is a direct target of valproic acid, a potent anticonvulsant, mood stabilizer, and teratogen. Journal of Biological Chemistry 2001, 276:36734-36741.

7. Blaheta RA, Cinatl J Jr: Anti-tumor mechanisms of valproate: a novel role for an old drug. Med Res Rev 2002, 22:492-511.

8. Huang C, Jacobson K, Schaller MD: MAP kinases and cell migration. Journal of Cell Science 2004, 117:4619-4628.

9. Meloche $S$, Pouyssegur J: The ERK $1 / 2$ mitogen-activated protein kinase pathway as a master regulator of the G1- to S-phase transition. Oncogene 2007, 26:3227-3239.

10. Yoon S, Seger R: The extracellular signal-regulated kinase: multiple substrates regulate diverse cellular functions. Growth Factors 2006, 24:21-44.

11. MCKay MM, Morrison DK: Integrating signals from RTKs to ERK/MAPK. Oncogene 2007, 26:3113-3121.

12. Dhanasekaran N, Premkumar Reddy E: Signaling by dual specificity kinases. Oncogene 1998, 17:1447-1455.

13. Hagemann C, Rapp UR: Isotype-specific functions of Raf kinases. Experimental Cell Research 1999, 253:34-46.

14. Rozengurt $E:$ Mitogenic signaling pathways induced by $\mathrm{G}$ proteincoupled receptors. J Cell Physiol 2007, 213:589-602.

15. Knupfer MM, Pulzer F, Schindler I, Hernaiz Driever P, Knupfer $H$, Keller E: Different effects of valproic acid on proliferation and migration of malignant glioma cells in vitro. Anticancer Res 2001, 21:347-351.

16. Witt O, Monkemeyer S, Kanbach K, Pekrun A: Induction of fetal hemoglobin synthesis by valproate: modulation of MAP kinase pathways. American Journal of Hematology 2002, 71:45-46.
17. Yuan PX, Huang LD, Jiang YM, Gutkind JS, Manji HK, Chen G: The mood stabilizer valproic acid activates mitogen-activated protein kinases and promotes neurite growth. Journal of Biological Chemistry 2001, 276:31674-31683.

18. Gotfryd K, Owczarek S, Hoffmann K, Klementiev B, Nau H, Berezin V, Bock E, Walmod PS: Multiple effects of pentyl-4-yn-VPA enantiomers: from toxicity to short-term memory enhancement. Neuropharmacology 2007, 52:764-778.

19. Walmod PS, Hartmann-Petersen R, Berezin A, Prag S, Kiselyov W, Berezin V, Bock E: Evaluation of individual-cell motility. Methods in Molecular Biology 2001, 161:59-83.

20. Pullikuth AK, Catling AD: Scaffold mediated regulation of MAPK signaling and cytoskeletal dynamics: a perspective. Cell Signal 2007, 19:1621-1632.

21. Walmod PS, Foley A, Berezin A, Ellerbeck U, Nau H, Bock E, Berezin V: Cell motility is inhibited by the antiepileptic compound, valproic acid and its teratogenic analogues. Cell Motilility and the Cytoskeleton 1998, 40:220-237.

22. Yamamoto T, Taya S, Kaibuchi K: Ras-Induced Transformation and Signaling Pathway. J Biochem 1999, 126:799-803.

23. Gottlicher M, Minucci S, Zhu P, Kramer OH, Schimpf A, Giavara S, Sleeman JP, Lo Coco F, Nervi C, Pelicci PG, Heinzel T: Valproic acid defines a novel class of HDAC inhibitors inducing differentiation of transformed cells. EMBO Journal 2001, 20:6969-6978.

24. Blaheta RA, Nau H, Michaelis M, Cinatl J Jr: Valproate and valproateanalogues: potent tools to fight against cancer. Curr Med Chem 2002, 9:1417-1433.

25. Camphausen K, Cerna D, Scott T, Sproull M, Burgan WE, Cerra MA, Fine H, Tofilon PJ: Enhancement of in vitro and in vivo tumor cell radiosensitivity by valproic acid. Int I Cancer 2005, 114:380-386.

26. Xia Q, Sung J, Chowdhury W, Chen CL, Hoti N, Shabbeer S, Carducci M, Rodriguez R: Chronic administration of valproic acid inhibits prostate cancer cell growth in vitro and in vivo. Cancer Res 2006, 66:7237-7244.

27. Hao Y, Creson T, Zhang L, Li P, Du F, Yuan P, Gould TD, Manji HK, Chen G: Mood stabilizer valproate promotes ERK pathway-dependent cortical neuronal growth and neurogenesis. J Neurosci 2004, 24:6590-6599.

28. Di Daniel E, Mudge AW, Maycox PR: Comparative analysis of the effects of four mood stabilizers in SH-SY5Y cells and in primary neurons. Bipolar Disord 2005, 7:33-41.

29. Michaelis M, Suhan T, Michaelis UR, Beek K, Rothweiler F, Tausch L, Werz O, Eikel D, Zornig M, Nau H, Fleming I, Doerr HW, Cinatl J Jr: Valproic acid induces extracellular signal-regulated kinase $1 / 2$ activation and inhibits apoptosis in endothelial cells. Cell Death Differ 2006, 13:446-453.

30. Vulcano F, Ciccarelli C, Mattia G, Marampon F, Giampiero M, Milazzo L, Pascuccio M, Zani BM, Giampaolo A, Hassan HJ: HDAC inhibition is associated to valproic acid induction of early megakaryocytic markers. Experimental Cell Research 2006, 312:1590-1597.

31. Jung JW, Cho SD, Ahn NS, Yang SR, Park JS, Jo EH, Hwang JW, Jung JY, Kim SH, Kang KS, Lee YS: Ras/MAP kinase pathways are involved in Ras specific apoptosis induced by sodium butyrate. Cancer Lett 2005, 225:199-206.

32. Yu C, Subler M, Rahmani M, Reese E, Krystal G, Conrad D, Dent P, Grant S: Induction of apoptosis in $B C R / A B L+$ cells by histone deacetylase inhibitors involves reciprocal effects on the RAF/MEK/ERK and JNK pathways. Cancer Biol Ther 2003, 2:544-551.

33. Michaelis M, Suhan $T$, Reinisch A, Reisenauer A, Fleckenstein C, Eikel D, Gumbel H, Doerr HW, Nau H, Cinatl J Jr: Increased replication of human cytomegalovirus in retinal pigment epithelial cells by valproic acid depends on histone deacetylase inhibition. Invest Ophthalmol Vis Sci 2005, 46:3451-3457.

34. Catalano MG, Poli R, Pugliese M, Fortunati N, Boccuzzi G: Valproic acid enhances tubulin acetylation and apoptotic activity of paclitaxel on anaplastic thyroid cancer cell lines. Endocr Relat Cancer 2007, 14:839-845.

35. Gurvich N, Tsygankova OM, Meinkoth JL, Klein PS: Histone deacetylase is a target of valproic acid-mediated cellular differentiation. Cancer Res 2004, 64:1079-1086.

36. Zhang X, Yuan Z, Zhang Y, Yong S, Salas-Burgos A, Koomen J, Olashaw N, Parsons JT, Yang XJ, Dent SR, Yao TP, Lane WS, Seto E: HDAC6 modulates cell motility by altering the acetylation level of cortactin. Mol Cell 2007, 27:197-213.

37. Boutros $T$, Chevet $E$, Metrakos P: Mitogen-activated protein (MAP) kinase/ MAP kinase phosphatase regulation: roles in cell growth, death, and cancer. Pharmacol Rev 2008, 60:261-310. 
38. Fuller LC, Cornelius SK, Murphy CW, Wiens DJ: Neural crest cell motility in valproic acid. Reproductive Toxicology 2002, 16:825-839.

39. Chen CL, Sung J, Cohen M, Chowdhury WH, Sachs MD, Li Y, Lakshmanan Y, Yung BY, Lupold SE, Rodriguez R: Valproic acid inhibits invasiveness in bladder cancer but not in prostate cancer cells. J Pharmacol Exp Ther 2006, 319:533-542.

40. De Girolamo LA, Billett EE: Role of extracellular-regulated kinase and c Jun NH2-terminal kinase in 1-methyl-4-phenyl-1,2,3,6-tetrahydropyridineinduced neurofilament phosphorylation. J Neurosci Res 2006, 83:680-693.

41. O'Neill E, Kolch W: Conferring specificity on the ubiquitous Raf/MEK signalling pathway. Br J Cancer 2004, 90:283-288.

42. Beeram M, Patnaik A, Rowinsky EK: Raf: a strategic target for therapeutic development against cancer. J Clin Oncol 2005, 23:6771-6790.

43. Das CM, Aguilera D, Vasquez H, Prasad P, Zhang M, Wolff JE, Gopalakrishnan V: Valproic acid induces p21 and topoisomerase-II (alpha/ beta) expression and synergistically enhances etoposide cytotoxicity in human glioblastoma cell lines. J Neurooncol 2007, 85:159-170.

\section{Pre-publication history}

The pre-publication history for this paper can be accessed here: http://www.biomedcentral.com/1471-2407/10/383/prepub

doi:10.1186/1471-2407-10-383

Cite this article as: Gotfryd et al:: Cell type-specific anti-cancer properties of valproic acid: independent effects on HDAC activity and Erk1/2 phosphorylation. BMC Cancer 2010 10:383.

\section{Submit your next manuscript to BioMed Central} and take full advantage of:

- Convenient online submission

- Thorough peer review

- No space constraints or color figure charges

- Immediate publication on acceptance

- Inclusion in PubMed, CAS, Scopus and Google Scholar

- Research which is freely available for redistribution

Submit your manuscript at www.biomedcentral.com/submit 